\title{
Automated Theorem Proving in Incidence Geometry - A Bracket Algebra Based Elimination Method
}

\author{
Hongbo Li and Yihong $\mathrm{Wu}$ \\ Institute of Systems Science, Academy of Mathematics and Systems Science \\ Chinese Academy of Sciences, Beijing 100080.
}

\begin{abstract}
In this paper we propose a bracket algebra based elimination method for automated generation of readable proofs for theorems in incidence geometry. This method is based on two techniques, the first being some heuristic elimination rules which improve the performance of the area method of Chou et al. (1994) without introducing signed length ratios, the second being a simplification technique called contraction, which reduces the size of bracket polynomials. More than twenty theorems in incidence geometry have been proved, for which short proofs are produced swiftly. An interesting phenomenon is that a proof composed of polynomials of at most two terms can always be found for any of these theorems, similar to that by the final biquadratic polynomial method of Richter-Gebert (1995).
\end{abstract}

\section{Introduction}

According to Richter-Gebert (1995), brackets are the fundamental invariants under projective transformations. From an invariant theoretic point of view, the ring of brackets forms a suitable algebraic setting to deal with projective configurations. The bracket algebra is the most general structure in which projective properties can be expressed in a coordinate-free way.

Let $\mathcal{V}^{n+1}$ be an $(n+1)$-dimensional vector space. For a sequence of $n+1$ vectors $\mathbf{A}_{1}, \ldots, \mathbf{A}_{n+1} \in \mathcal{V}^{n+1}$, the corresponding bracket is defined by

$$
\left[\mathbf{A}_{1} \cdots \mathbf{A}_{n+1}\right]=\operatorname{det}\left(\mathbf{A}_{1} \cdots \mathbf{A}_{n+1}\right) .
$$

Let $\mathbf{A}_{1}, \ldots, \mathbf{A}_{m}$ be indeterminates (vectors) in $\mathcal{V}^{n+1}, m>n$. The bracket algebra generated by them is the polynomial algebra $\mathcal{R}\left(\left[\mathbf{A}_{i_{1}} \cdots \mathbf{A}_{i_{n+1}}\right] \mid 1 \leq i_{j} \leq m\right)$ generated by all possible brackets of the indeterminates modulo the ideal generated by the following GrassmannPlücker polynomials:

$$
\begin{aligned}
& \mathcal{G P}=\left\{\sum_{k=1}^{n+2}(-1)^{k}\left[\mathbf{A}_{i_{1}} \cdots \mathbf{A}_{i_{n}} \mathbf{A}_{j_{k}}\right]\left[\mathbf{A}_{j_{1}} \cdots \mathbf{A}_{j_{k-1}} \mathbf{A}_{j_{k+1}} \cdots \mathbf{A}_{j_{n+2}}\right]\right. \\
& \left.\mid 1 \leq i_{1}<\cdots<i_{n} \leq m, 1 \leq j_{1}<\cdots<j_{n+2} \leq m\right\} .
\end{aligned}
$$

On the level of bracket algebra, a geometric theorem prover can be implemented using the straightening algorithm (Young, 1928; Doubilet et al., 1974). The main idea behind this approach is to rewrite the projective incidence statement as a term in Grassmann algebra 
which vanishes if and only if the statement is true. After this, the Grassmann algebra term is expanded into a bracket one. If this term vanishes modulo the ideal generated by the Grassmann-Plücker polynomials, then the theorem is proved. It is proved by Sturmfels and White (1989) that the straightening algorithm can be considered as a special kind of Gröbner bases algorithm for bracket polynomials. The algorithm works in full generality, but requires over-exponential CPU time.

The prover proposed by Richter-Gebert (1995) is based on the final biquadratic polynomial method (see also Bokowski and Richter-Gebert, 1990; Sturmfels, 1989). A proof produced by this prover is extremely short and geometrically meaningful. In particular, every polynomial occurred in the proof is composed of two terms. Although the algorithm does not work in general, it could manage almost all projective incidence theorems.

Another prover is proposed by Chou et al. (1994) and is based on the area method. This is an elimination method whose rules are derived from properties of signed areas, or brackets in 2-d projective space. This method is complete when area coordinates are used. When the coordinates are avoided, proofs produced by the prover are often short and readable.

Our work is inspired both by the area method and by the final polynomial method. First, we propose a set of heuristic elimination rules to improve the performance of the area method by producing shorter proofs. Second, we propose a new technique for bracket polynomial simplification, a special case of which is used as the foundation for setting up biquadratic equations in the final polynomial method. We build up a prover based on the two techniques.

The performance of the prover is very satisfactory: more than twenty incidence theorems have been tested, which covers all the 2-d incidence theorems in (Chou et al., 1994) and (Richter-Gebert, 1995). For every theorem, a proof composed of polynomials of at most two terms can be produced very fast. Furthermore, every proof finishes before any free point in the plane is eliminated, and in some cases, even before some semifree points on lines are eliminated.

The prover is complete for 2 -d incidence theorems of the following constructive types.

Constructive type $\mathbf{1}$. Take a free point in the plane.

Constructive type 2. Take a semifree point on a line.

Constructive type 3. Take the intersection of two lines.

\section{Algorithm}

The following is an algorithm which can produce a proof in the form of brackets for a theorem whose conclusion is either an equality or an inequality.

\section{Input.}

- A set of constructions of points.

- An order for eliminations of points.

- A conclusion $c o n c=0$ or $c o n c \neq 0$, where conc is a polynomial of brackets.

Preprocess. Change every collinearity constraint in the constructions into a rule for computing brackets. 
Step 1. Eliminate constrained points and semifree points. First, assume that point $\mathbf{X}$ is the intersection of lines $\mathbf{A B}$ and $\mathbf{C D}$. To eliminate $\mathbf{X}$ from a bracket $[\mathbf{X P Q}$, there are three formulas available:

$$
\begin{aligned}
& {[\mathbf{X P Q}]=\mathbf{X} \vee(\mathbf{P} \wedge \mathbf{Q})=(\mathbf{A} \wedge \mathbf{B}) \vee(\mathbf{C} \wedge \mathbf{D}) \vee(\mathbf{P} \wedge \mathbf{Q})} \\
& \quad= \begin{cases}{[\mathbf{A B D}][\mathbf{C P Q}]-[\mathbf{A B C}][\mathbf{D P Q}]} \\
{[\mathbf{A C D}][\mathbf{B P Q}]-[\mathbf{B C D}][\mathbf{A P Q}]} \\
{[\mathbf{A B P}][\mathbf{C D Q}]-[\mathbf{A B Q}][\mathbf{C D P}]}\end{cases}
\end{aligned}
$$

Rule 1. (same as in the area method) If a bracket in (??) equals zero, use the corresponding formula.

Rule 2. (heuristic) In general, use the formula which separates into different brackets the pair of points in $(\mathbf{A}, \mathbf{B}),(\mathbf{C}, \mathbf{D}),(\mathbf{P}, \mathbf{Q})$ having the largest number of concurrent lines.

In the area method, (??.2) is generally adopted.

Second, assume that point $\mathbf{X}$ is on line $\mathbf{A B}$. Let $\mathbf{A}, \mathbf{B}, \mathbf{C}$ be linearly independent vectors. To eliminate $\mathbf{X}$ from a bracket polynomial $p$, first contract $p$ (see Step 2), then for each $[\mathbf{X P Q}]$ in $p$, apply the following formula:

$$
[\mathbf{A B C}][\mathbf{X P Q}]=[\mathbf{X B C}][\mathbf{A P Q}]-[\mathbf{X A C}][\mathbf{B P Q}],
$$

which is a Grassmann-Plücker relation in the case $[\mathbf{X A B}]=0$.

Rule 3. (heuristic) In general, choose $\mathbf{C}$ to be the free point outside line $\mathbf{A B}$ that has the largest number of occurrences in $p$. The nondegeneracy condition is $[\mathbf{A B C}] \neq 0$ if $[\mathbf{A B C}]$ occurs in the denominator of $p$.

Step 2. Simplification by contraction. For any vectors $\mathbf{A}_{1}, \ldots, \mathbf{A}_{5}$ in $\mathcal{R}^{3}$,

$$
\left[\mathbf{A}_{1} \mathbf{A}_{2} \mathbf{A}_{5}\right]\left[\mathbf{A}_{3} \mathbf{A}_{4} \mathbf{A}_{5}\right]+\left[\mathbf{A}_{1} \mathbf{A}_{3} \mathbf{A}_{5}\right]\left[\mathbf{A}_{4} \mathbf{A}_{2} \mathbf{A}_{5}\right]=\left[\mathbf{A}_{1} \mathbf{A}_{4} \mathbf{A}_{5}\right]\left[\mathbf{A}_{3} \mathbf{A}_{2} \mathbf{A}_{5}\right] \text {. }
$$

This is a Grassmann-Plücker relation.

Let $p$ be a bracket polynomial of two terms. If $p$ is reduced to a single monomial by the above identity, this reduction is called a contraction. It can be extended to any bracket polynomial.

It can be proved that a polynomial is reduced to zero modulo the ideal generated by the Grassmann-Plücker polynomials if and only if when multiplied by some bracket polynomial, it is reduced to zero through contractions. As a result, the outcome of the contraction is always zero for $c o n c=0$, and nonzero for $c o n c \neq 0$.

Output. The proving procedure and the nondegeneracy conditions.

Remark 1. The heuristic rules Rule 2 and Rule 3 can contribute to obtaining short proofs. When searching for a proof composed of polynomials of at most two terms, these rules can serve as guidelines for setting up precedence tables.

Remark 2. To improve the performance of the algorithm for conc $=0$, after each elimination we can delete the common bracket factors in conc. These factors are not nondegeneracy conditions. 


\section{Examples}

Below is a collection of 23 examples and their machine generated proofs composed of polynomials of at most two terms. The program is written in Maple V.4 and runs on an IBM compatible Pentium II/366 with Microsoft Windows 98. The generation of each proof is very fast. The nondegeneracy conditions are generated at the same time.

For theorems of equality type, common bracket factors (underlined) are found out in each step and are deleted before the next step starts.

Example 1. [See also Chou et. al. (1994), Example 6.203]

Free points: 1, 2, 3, 4 .

Intersections:

$$
\begin{array}{lll}
5=12 \cap 34, & 6=13 \cap 24, & 7=23 \cap 14, \\
8=23 \cap 56, & 9=24 \cap 57, & 0=34 \cap 67 .
\end{array}
$$

Conclusion: 8, 9, 0 are collinear.

Fig. 1. Example 1.

\begin{tabular}{|c|c|c|c|}
\hline \multicolumn{2}{|r|}{ Rules } & & {$[890]$} \\
\hline $\begin{array}{l}{[689]} \\
{[789]}\end{array}$ & $\begin{array}{l}=[\mathbf{2 4 8}][567] \\
=[247][578]\end{array}$ & $\underline{\underline{9}}$ & {$[248][347][567]-[247][346][578]$} \\
\hline $\begin{array}{l}{[248]} \\
{[578]}\end{array}$ & $\begin{array}{l}=[\mathbf{2 3 6}][\mathbf{2 4 5}] \\
=\quad[\mathbf{2 3 5}][567]\end{array}$ & $\underline{\underline{8}}$ & {$[567][236][245][347]-[567][235][247][346]$} \\
\hline $\begin{array}{l}{[347]} \\
{[247]}\end{array}$ & $\begin{array}{l}=[134][234] \\
=\quad[124][234]\end{array}$ & $\stackrel{7}{=}$ & {$[234][134][245][236]-[234][124][235][346]$} \\
\hline $\begin{array}{l}{[236]} \\
{[346]}\end{array}$ & $\begin{array}{l}=[123][234] \\
=[134][234]\end{array}$ & $\underline{\underline{6}}$ & $\underline{[134][234]}([123][245]-[124][235])$ \\
\hline $\begin{array}{l}{[245]} \\
{[235]}\end{array}$ & $\begin{array}{ll}= & -[124][234] \\
= & -[123][234]\end{array}$ & $\underline{\underline{5}}$ & 0. \\
\hline
\end{tabular}

Proof:

Nondegeneracy condition: none. 
Example 2. [See also Kadison and Kromann (1996), Proposition 5.8]

Free points: 1, 2, 3, 4 .

Intersections:

$$
\begin{array}{lll}
5=12 \cap 34, & 6=13 \cap 24, & 7=23 \cap 14, \\
8=13 \cap 57, & 9=67 \cap 48, & 0=24 \cap 57 .
\end{array}
$$

Conclusion: $\mathbf{3 ,} \mathbf{9}, \mathbf{0}$ are collinear.

Fig. 2. Example 2.

\begin{tabular}{|c|c|c|c|}
\hline \multirow{2}{*}{\multicolumn{2}{|c|}{ Rules }} & \multirow[b]{2}{*}{$\underline{\underline{0}}$} & [390] \\
\hline & & & $-[257][349]-[239][457]$ \\
\hline $\begin{array}{l}{[349]} \\
{[239]}\end{array}$ & $\begin{array}{rr}= & -[348][467] \\
= & {[236][478]}\end{array}$ & $\underline{\underline{9}}$ & {$[257][348][467]-[236][457][478]$} \\
\hline $\begin{array}{l}{[348]} \\
{[478]}\end{array}$ & $\begin{array}{l}=-[134][357] \\
=-[137][457]\end{array}$ & $\underline{\underline{8}}$ & $-[134][257][357][467]+[137][236][457]^{2}$ \\
\hline$[257]$ & $=\quad[124][235]$ & & \\
\hline [467] & $=\quad[146][234]$ & & \\
\hline [357] & {$[134][235]$} & $\stackrel{\rho}{=}$ & {$[134][234]\left(-[124][134][146][235]^{2}-[123][145]^{2}[234][236]\right.$} \\
\hline [457] & $=[145][234]$ & & \\
\hline [137] & $=-[\mathbf{1 2 3}][\mathbf{1 3 4}]$ & & \\
\hline $\begin{array}{l}{[146]} \\
{[236]}\end{array}$ & $\begin{array}{rr}= & -[124][134] \\
= & {[123][234]}\end{array}$ & $\underline{\underline{6}}$ & {$[\mathbf{1 2 4}]^{2}[\mathbf{1 3 4}]^{2}[\mathbf{2 3 5}]^{2}-[\mathbf{1 2 3}]^{2}[\mathbf{1 4 5}]^{2}[\mathbf{2 3 4}]^{2}$} \\
\hline [235] & $=-[123][234]$ & $\underline{\underline{\mathbf{5}}}$ & 0 \\
\hline [145] & $=-[124][134]$ & & \\
\hline
\end{tabular}

Proof:

Nondegeneracy condition: none.

Example 3. [See also Pedoe (1963), p. 63]

Free points: 1, 2, 3, 4.

Intersections:

$$
\begin{array}{lll}
5=12 \cap 34, & 6=13 \cap 24, & 7=23 \cap 14, \\
8=13 \cap 57, & 9=14 \cap 56, & 0=34 \cap 67 .
\end{array}
$$


Conclusion: $\mathbf{8 , ~ 9 , ~} \mathbf{0}$ are collinear.

Fig. 3. Example 3.

Proof:

\begin{tabular}{|c|c|c|c|}
\hline \multicolumn{2}{|r|}{ Rules } & & {$[890]$} \\
\hline $\begin{array}{l}{[689]} \\
{[789]}\end{array}$ & $\begin{array}{l}=[156][468] \\
=-[148][567]\end{array}$ & $\underline{\underline{9}}$ & {$[156][347][468]+[148][346][567]$} \\
\hline $\begin{array}{l}{[468]} \\
{[148]}\end{array}$ & $\begin{array}{l}=-[\mathbf{1 3 4}][\mathbf{5 6 7}] \\
=-[\mathbf{1 3 4}][\mathbf{1 5 7}]\end{array}$ & $\underline{\underline{8}}$ & $\underline{[134][567]}(-[156][347]-[157][346])$ \\
\hline $\begin{array}{l}{[347]} \\
{[157]}\end{array}$ & $\begin{array}{l}=[\mathbf{1 3 4}][\mathbf{2 3 4}] \\
=[\mathbf{1 2 3}][\mathbf{1 4 5}]\end{array}$ & $\stackrel{7}{=}$ & $-[134][156][234]-[123][145][346]$ \\
\hline $\begin{array}{l}{[156]} \\
{[346]}\end{array}$ & $\begin{aligned}= & -[\mathbf{1 2 4}][\mathbf{1 3 5}] \\
= & {[\mathbf{1 3 4}][\mathbf{2 3 4}] }\end{aligned}$ & $\underline{\underline{6}}$ & $\underline{[134][234]}([\mathbf{1 2 4}][135]-[123][145])$ \\
\hline $\begin{array}{l}{[135]} \\
{[145]}\end{array}$ & $\begin{array}{l}=-[\mathbf{1 2 3}][\mathbf{1 3 4}] \\
=-[\mathbf{1 2 4}][\mathbf{1 3 4}]\end{array}$ & $\underline{\underline{5}}$ & 0. \\
\hline
\end{tabular}

Nondegeneracy condition: none.

Example 4. [See also Chou et. al. (1994), Example 6.32]

Free points: $1,2,3,4,5$.

Intersections:

$$
\begin{aligned}
& 6=12 \cap 34, \quad 7=13 \cap 24, \quad 8=23 \cap 14, \quad 9=56 \cap 78, \\
& 0=57 \cap 68, \quad A=39 \cap 20, \quad B=67 \cap 58 .
\end{aligned}
$$

Conclusion: 1, A, $\mathbf{B}$ are collinear. 
Fig. 4. Example 4.

Proof:

\begin{tabular}{|c|c|c|c|}
\hline \multicolumn{2}{|r|}{ Rules } & \multicolumn{2}{|r|}{$[\mathbf{1 A B}]$} \\
\hline $\begin{array}{l}{[\mathbf{1 7} \mathbf{A}]} \\
{[16 \mathbf{A}]}\end{array}$ & $\begin{array}{l}=-[\mathbf{1 7 9}][\mathbf{2 3 0}] \\
=-[\mathbf{1 6 0}][\mathbf{2 3 9}]\end{array}$ & $\stackrel{\mathrm{A}}{=}$ & $-[179][230][568]+[160][239][578]$ \\
\hline $\begin{array}{l}{[230]} \\
{[160]}\end{array}$ & $\begin{array}{l}=[\mathbf{2 3 6}][578] \\
=[168][567]\end{array}$ & $\underline{\underline{0}}$ & $-\underline{[578}][179][236][568]+\underline{[578]}[168][239][567$ \\
\hline $\begin{array}{l}{[\mathbf{1 7 9}]} \\
{[239]}\end{array}$ & $\begin{array}{l}=-[\mathbf{1 7 8}][567] \\
=\quad[237][568]\end{array}$ & $\underline{9}$ & $\underline{[567][568]}([178][236]+[168][237])$ \\
\hline $\begin{array}{l}{[178]} \\
{[168]}\end{array}$ & $\begin{array}{l}=[123][147] \\
=[123][146]\end{array}$ & $\underline{=}$ & $\underline{[123]}[147][236]+\underline{[123]}[146][237]$ \\
\hline $\begin{array}{l}{[\mathbf{1 4 7}]} \\
{[237]}\end{array}$ & $\begin{array}{l}=-[\mathbf{1 2 4}][134] \\
=\quad[123][234]\end{array}$ & $\underline{7}$ & $-[\mathbf{1 2 4}][\mathbf{1 3 4}][\mathbf{2 3 6}]+[\mathbf{1 2 3}][\mathbf{1 4 6}][\mathbf{2 3 4}]$ \\
\hline $\begin{array}{l}{[236]} \\
{[146]}\end{array}$ & $\begin{array}{l}=-[\mathbf{1 2 3}][\mathbf{2 3 4}] \\
=-[\mathbf{1 2 4}][\mathbf{1 3 4}]\end{array}$ & $\underline{\underline{6}}$ & 0. \\
\hline
\end{tabular}

Nondegeneracy condition: none.

Example 5. [Pappus point theorem, see also Chou et. al. (1994), Example 6.22]

Free points: $\mathbf{1}, \mathbf{2}, \mathbf{3}, \mathbf{4}, \mathbf{5}$.

Intersections:

$$
\begin{aligned}
& 6=13 \cap 24, \quad 7=23 \cap 56, \quad 8=25 \cap 34, \quad 9=12 \cap 68, \\
& 0=79 \cap 24, \quad \mathrm{~A}=39 \cap 67, \quad \mathrm{~B}=15 \cap 4 \mathrm{~A}, \quad \mathrm{C}=28 \cap 39 .
\end{aligned}
$$

Conclusion: $\mathbf{0}, \mathbf{B}, \mathrm{C}$ are collinear. 
Fig. 5. Example 5.

Proof:

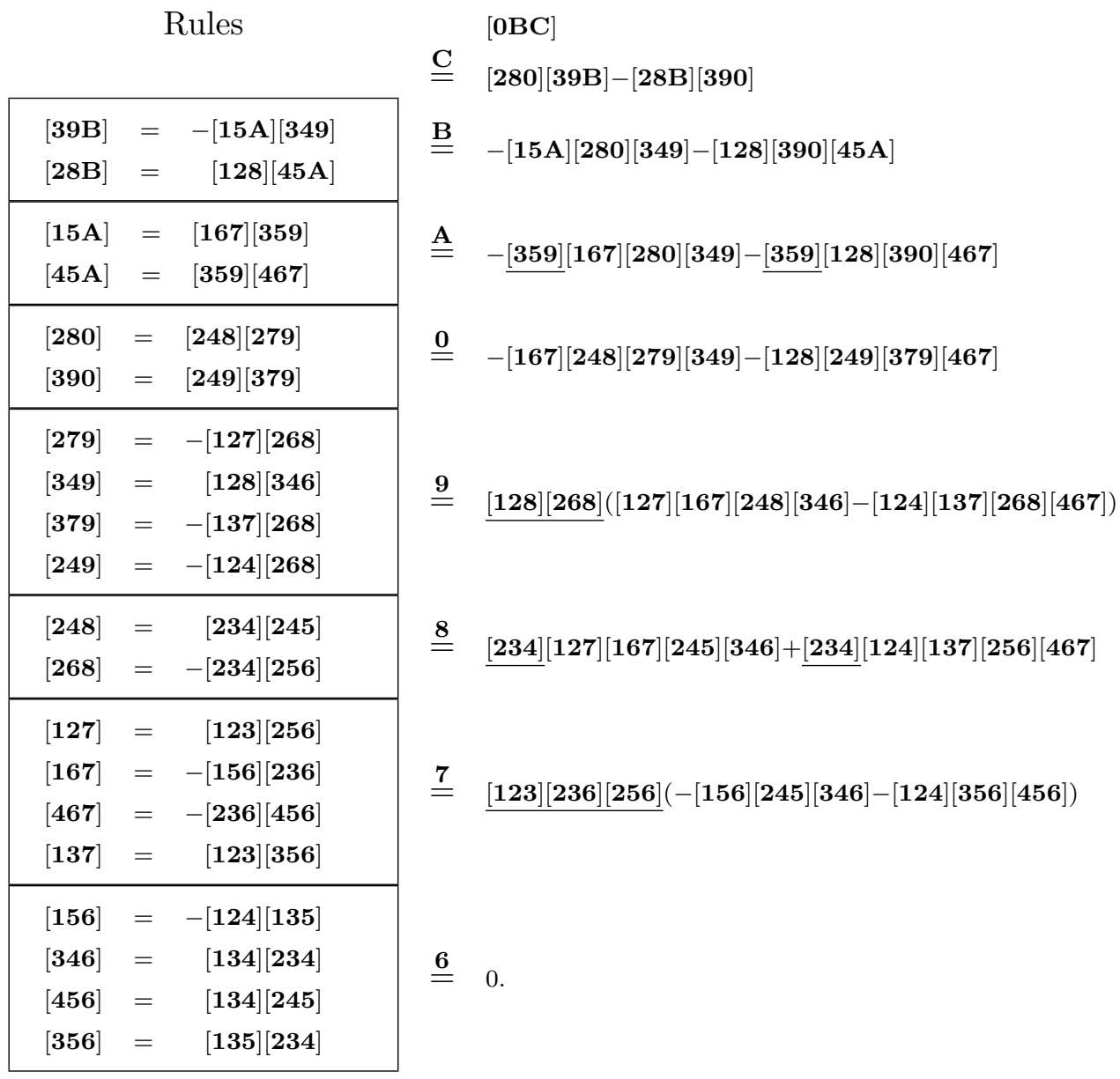

Nondegeneracy condition: none. 
Example 6. [Pappus Theorem, see also Chou et. al. (1994), Example 6.20]

Free points: $1, \mathbf{2}, \mathbf{3}, \mathbf{4}$.

Semifree points: $\mathbf{5}$ on $\mathbf{1 2}, \mathbf{6}$ on $\mathbf{3 4}$.

Intersections: $7=23 \cap 14,8=35 \cap 16,9=45 \cap 26$.

Conclusion: $\mathbf{7 ,} 8, \mathbf{9}$ are collinear.

Fig. 6. Example 6 .

Proof:

\begin{tabular}{|c|c|c|c|}
\hline & Rules & & {$[\mathbf{7 8 9}]$} \\
\hline $\begin{array}{l}{[\mathbf{2 7 8}]=} \\
{[\mathbf{6 7 8}]=}\end{array}$ & $\begin{array}{l}{[\mathbf{1 3 6}][\mathbf{2 5 7}]} \\
{[\mathbf{1 6 7}][\mathbf{3 5 6}]}\end{array}$ & $\underline{\underline{8}}$ & {$[136][257][456]-[167][245][356]$} \\
\hline $\begin{array}{l}{[\mathbf{2 5 7}]=} \\
{[\mathbf{1 6 7}]=}\end{array}$ & $\begin{array}{l}{[\mathbf{1 2 4}][\mathbf{2 3 5}]} \\
{[\mathbf{1 2 3}][\mathbf{1 4 6}]}\end{array}$ & $\underline{7}$ & {$[124][136][235][456]-[123][146][245][356]$} \\
\hline $\begin{array}{l}{[134][456} \\
{[134][356}\end{array}$ & $\begin{array}{l}=-[\mathbf{1 4 6}][\mathbf{3 4 5}] \\
=-[\mathbf{1 3 6}][\mathbf{3 4 5}]\end{array}$ & $\underline{6}$ & $\frac{[136][146][345]}{[[134]}(-[124][235]+[123][245])$ \\
\hline
\end{tabular}

Nondegeneracy condition: $[\mathbf{1 3 4}] \neq 0$.

Example 7. [Desargues Theorem, see also Chou et. al. (1994), Example 6.24]

Free points: $\mathbf{1}, \mathbf{2}, \mathbf{3}, \mathbf{4}, \mathbf{5}$.

Semifree point: $\mathbf{6}$ on $\mathbf{1 3}$.

Intersections: $7=12 \cap 45, \quad 8=15 \cap 24, \quad 9=38 \cap 56, \quad 0=23 \cap 49$.

Conclusion: 6, 7, $\mathbf{0}$ are collinear. 
Fig. 7. Example 7.

Proof:

\begin{tabular}{|c|c|c|c|}
\hline \multicolumn{2}{|c|}{ Rules } & & {$[670]$} \\
\hline $\begin{array}{l}{[239]} \\
{[679]}\end{array}$ & $\begin{array}{rr}= & -[238][356] \\
= & {[368][567]}\end{array}$ & $\underline{\underline{9}}$ & $-[238][356][467]-[234][368][567]$ \\
\hline $\begin{array}{l}{[238]} \\
{[368]}\end{array}$ & $\begin{array}{rr}= & -[125][234] \\
= & {[124][356]}\end{array}$ & $\underline{\underline{8}}$ & $\underline{[234][356]}([125][467]-[124][567])$ \\
\hline $\begin{array}{l}{[467]} \\
{[567]}\end{array}$ & $\begin{array}{l}=[124][456] \\
=[125][456]\end{array}$ & $\stackrel{7}{=}$ & 0. \\
\hline
\end{tabular}

Nondegeneracy condition: none.

Example 8. [See also Chou et. al. (1994), Example 6.34]

Free points: 1, 2, 3 .

Semifree points: 4 on 12, 5 on 12, 6 on $\mathbf{1 3}, \mathbf{7}$ on 23.

Intersections:

$$
8=23 \cap 46, \quad 9=23 \cap 56, \quad 0=13 \cap 57, \quad A=13 \cap 47, \quad B=12 \cap 80 .
$$

Conclusion: 9, A, B are collinear.

Proof: 
Fig. 8. Example 8.

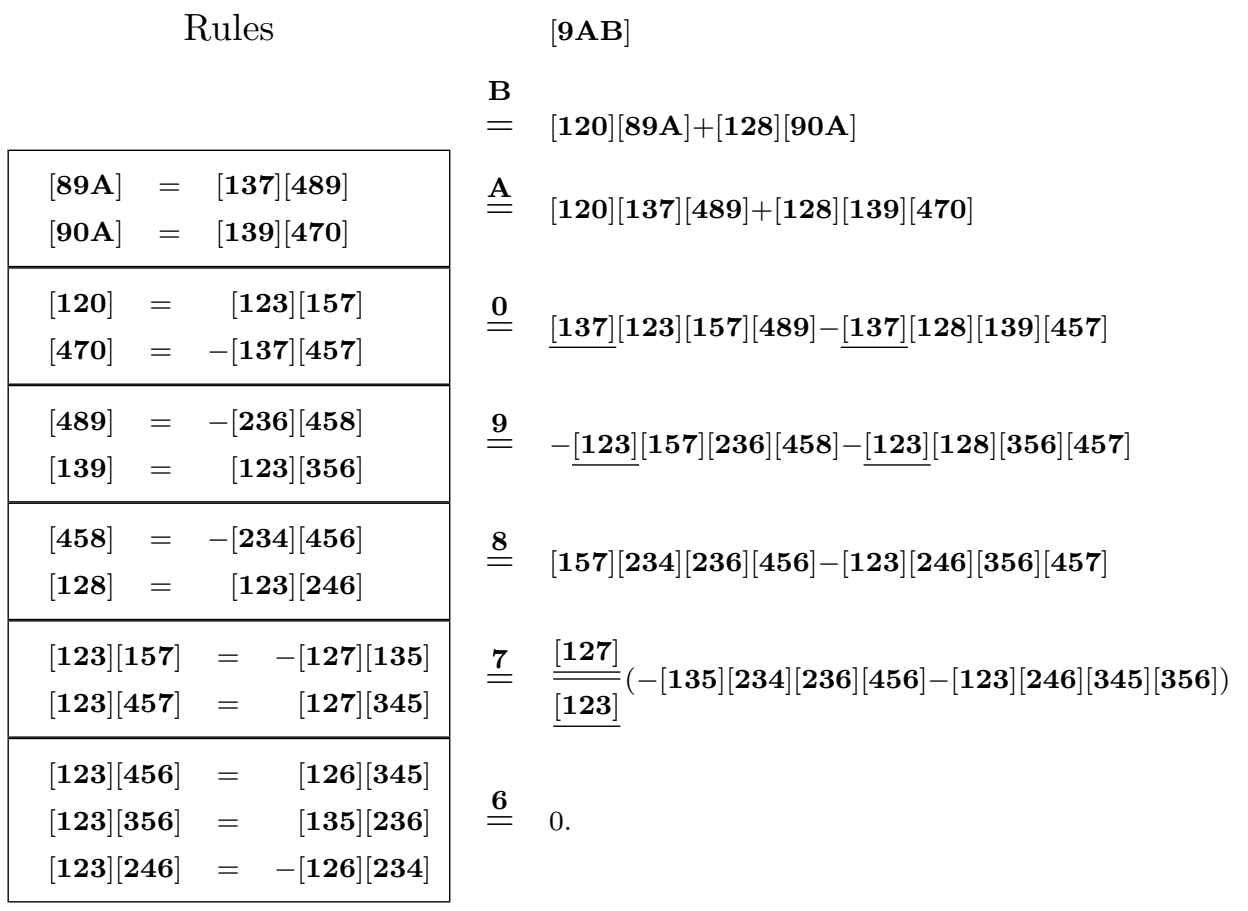

Nondegeneracy condition: $[\mathbf{1 2 3}] \neq 0$.

Example 9. [See also Chou et. al. (1994), Example 6.38]

Free points: 1, 2, 3, 4 .

Semifree point: $\mathbf{5}$ on $\mathbf{1 2}$.

Intersections:

$$
\begin{array}{lllll}
6=12 \cap 34, & 7=13 \cap 24, & 8=23 \cap 14, & 9=13 \cap 45, & 0=23 \cap 45, \\
\mathrm{~A}=14 \cap 35, & \mathrm{~B}=24 \cap 35, & \mathrm{C}=12 \cap 89, & \mathrm{D}=12 \cap 70, & \mathbf{E}=12 \cap 0 \mathrm{~A} .
\end{array}
$$


Conclusions: (1) $\mathbf{7}, \mathbf{A}, \mathbf{C}$ are collinear; (2) $\mathbf{8}, \mathbf{B}, \mathbf{D}$ are collinear; (3) $\mathbf{9}, \mathbf{B}, \mathbf{E}$ are collinear.

Fig. 9. Example 9.

Proof: (1)

\begin{tabular}{|c|c|c|c|}
\hline \multicolumn{2}{|r|}{ Rules } & & {$[\mathbf{7 A C}]$} \\
\hline $\begin{array}{l}{[27 \mathrm{~A}]} \\
{[17 \mathrm{~A}]}\end{array}$ & $\begin{array}{lr}= & {[127][\mathbf{3 4 5}]} \\
= & -[135][147]\end{array}$ & $\underline{\underline{A}}$ & {$[127][189][345]+[135][147][289]$} \\
\hline $\begin{array}{l}{[189]} \\
{[289]}\end{array}$ & $\begin{array}{l}=-[138][145] \\
=\quad-[128][345]\end{array}$ & $\underline{\underline{9}}$ & $-[345][127][138][145]-[345][128][135][147]$ \\
\hline $\begin{array}{l}{[138]} \\
{[128]}\end{array}$ & $\begin{array}{ll}= & -[123][134] \\
= & -[123][124]\end{array}$ & $\underline{\underline{8}}$ & $\underline{[123]}[127][134][145]+\underline{[123]}[124][135][147]$ \\
\hline $\begin{array}{l}{[127]} \\
{[147]}\end{array}$ & $\begin{array}{lr}= & {[123][124]} \\
= & -[124][134]\end{array}$ & $\underline{\underline{7}}$ & $\underline{[124][134]}([123][145]-[124][135])$ \\
\hline
\end{tabular}


$(2)$

\begin{tabular}{|c|c|c|c|c|}
\hline \multicolumn{3}{|c|}{ Rules } & & {$[8 \mathrm{BD}]$} \\
\hline $\begin{array}{l}{[28 \mathrm{~B}]} \\
{[18 \mathrm{~B}]}\end{array}$ & & $\begin{array}{l}-[\mathbf{2 3 5}][\mathbf{2 4 8}] \\
-[\mathbf{1 2 8}][\mathbf{3 4 5}]\end{array}$ & $\underline{\underline{B}}$ & $-[\mathbf{1 7 0}][\mathbf{2 3 5}][\mathbf{2 4 8}]+[\mathbf{1 2 8}][\mathbf{2 7 0}][\mathbf{3 4 5}]$ \\
\hline $\begin{array}{l}{[\mathbf{1 7 0}]} \\
{[\mathbf{2 7 0}]}\end{array}$ & & $\begin{array}{r}{[127][345]} \\
-[237][245]\end{array}$ & $\underline{\underline{0}}$ & $-\underline{[345]}[127][235][248]-\underline{[345]}[128][237][245]$ \\
\hline $\begin{array}{l}{[248]} \\
{[128]}\end{array}$ & $\begin{array}{l}= \\
=\end{array}$ & $\begin{array}{l}-[124][234] \\
-[123][124]\end{array}$ & $\underline{\underline{8}}$ & $\underline{[124]}[\mathbf{1 2 7}][\mathbf{2 3 4}][\mathbf{2 3 5}]+\underline{[\mathbf{1 2 4}]}[\mathbf{1 2 3}][\mathbf{2 3 7}][\mathbf{2 4 5}]$ \\
\hline $\begin{array}{l}{[127]} \\
{[237]}\end{array}$ & & $\begin{array}{r}-[123][124] \\
{[123][234]}\end{array}$ & $\stackrel{7}{=}$ & $\underline{[123][234]}(-[\mathbf{1 2 4}][\mathbf{2 3 5}]+[\mathbf{1 2 3}][\mathbf{2 4 5}])$ \\
\hline
\end{tabular}

$(3)$

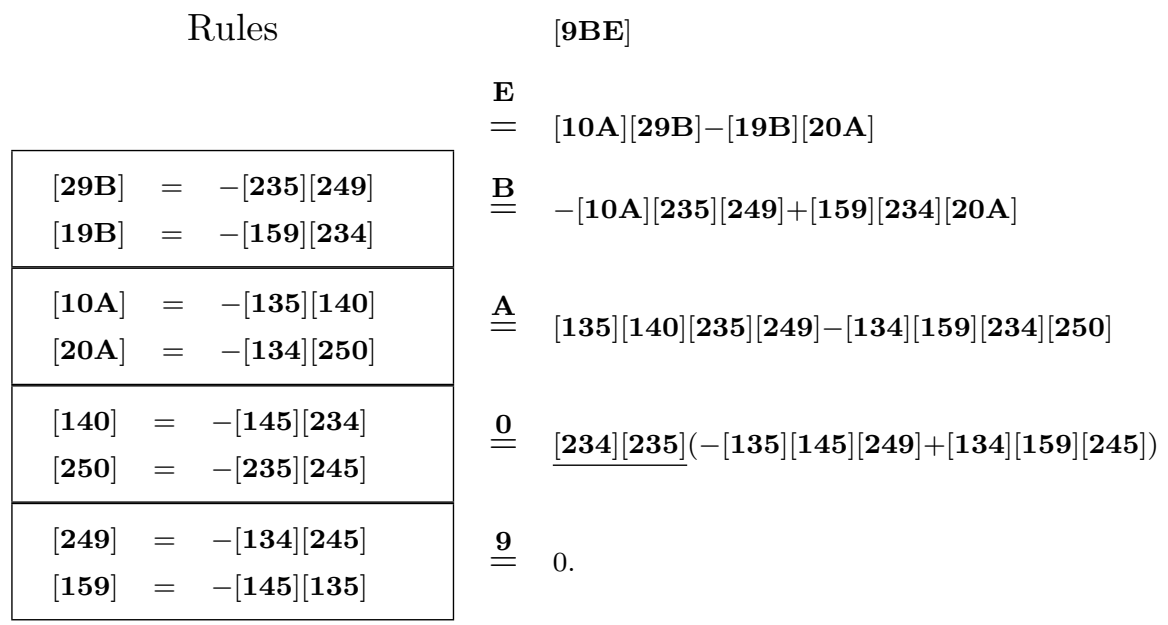

Nondegeneracy condition: none.

Example 10. [See also Chou et. al. (1994), Example 6.208]

Free points: 1, 2, 3, 4 .

Semifree point: $\mathbf{5}$ on $\mathbf{1 2}$.

Intersections:

$$
\begin{aligned}
& 6=12 \cap 34, \quad 7=13 \cap 24, \quad 8=13 \cap 45, \quad 9=23 \cap 67, \\
& 0=24 \cap 19, \quad A=34 \cap 19, \quad B=23 \cap 80, \quad C=49 \cap 30 .
\end{aligned}
$$

Conclusions: (1) 5, A, B are collinear; (2) $\mathbf{7}, \mathbf{A}, \mathbf{C}$ are collinear. 
Fig. 10. Example 10.

Proof: (1)

\begin{tabular}{|c|c|c|c|c|}
\hline \multicolumn{3}{|c|}{ Rules } & & {$[\mathbf{5 A B}]$} \\
\hline $\begin{array}{l}{[35 \mathbf{A}]} \\
{[25 \mathbf{A}]}\end{array}$ & & $\begin{array}{r}{[139][345]} \\
-[134][259]\end{array}$ & $\underline{\mathbf{A}}$ & {$[\mathbf{1 3 9}][\mathbf{2 8 0}][\mathbf{3 4 5}]+[\mathbf{1 3 4}][\mathbf{2 5 9}][\mathbf{3 8 0}]$} \\
\hline $\begin{array}{l}{[\mathbf{2 8 0}]} \\
{[\mathbf{3 8 0}]}\end{array}$ & $\begin{array}{l}= \\
=\end{array}$ & $\begin{array}{r}{[129][248]} \\
-[124][389]\end{array}$ & $\underline{\underline{0}}$ & {$[129][139][248][345]-[124][134][259][389]$} \\
\hline $\begin{array}{l}{[\mathbf{1 2 9}]} \\
{[139]} \\
{[\mathbf{3 8 9}]} \\
{[\mathbf{2 5 9}]}\end{array}$ & $\begin{array}{l}= \\
= \\
= \\
=\end{array}$ & $\begin{array}{r}{[123][267]} \\
{[123][367]} \\
-[238][367] \\
-[235][267]\end{array}$ & $\underline{\underline{9}}$ & {$[\mathbf{2 6 7}][367]\left([123]^{2}[248][345]-[124][134][235][238]\right)$} \\
\hline $\begin{array}{l}{[248]} \\
{[238]}\end{array}$ & $\begin{array}{l}= \\
=\end{array}$ & $\begin{array}{l}-[134][245] \\
-[123][345]\end{array}$ & $\underline{8}$ & $\underline{[\mathbf{1 2 3}][\mathbf{1 3 4}][\mathbf{3 4 5}]}(-[\mathbf{1 2 3}][\mathbf{2 4 5}]+[\mathbf{1 2 4}][\mathbf{2 3 5}])$ \\
\hline
\end{tabular}


$(2)$

\begin{tabular}{|c|c|c|c|}
\hline \multicolumn{2}{|r|}{ Rules } & & {$[\mathbf{7 A C}]$} \\
\hline $\begin{array}{l}{[79 \mathrm{~A}]} \\
{[47 \mathrm{~A}]}\end{array}$ & $\begin{array}{l}=[179][349] \\
=\quad[149][347]\end{array}$ & $\underline{\underline{A}}$ & {$[179][349][340]+[149][347][390]$} \\
\hline $\begin{array}{l}{[340]} \\
{[390]}\end{array}$ & $\begin{array}{l}=\quad[149][234] \\
=\quad[139][249]\end{array}$ & $\underline{\underline{0}}$ & $\underline{[149]}[179][234][349]+\underline{[149]}[139][249][347]$ \\
\hline $\begin{array}{l}{[179]} \\
{[349]} \\
{[249]} \\
{[139]}\end{array}$ & $\begin{array}{rr}= & -[167][237] \\
= & -[234][367] \\
= & -[234][267] \\
= & {[123][367]}\end{array}$ & $\underline{9}$ & $\underline{[\mathbf{2 3 4}][\mathbf{3 6 7}]}([\mathbf{1 6 7}][\mathbf{2 3 4}][\mathbf{2 3 7}]-[\mathbf{1 2 3}][\mathbf{2 6 7}][\mathbf{3 4 7}])$ \\
\hline $\begin{array}{l}{[237]} \\
{[167]} \\
{[347]} \\
{[267]}\end{array}$ & $\begin{array}{rr}= & {[123][234]} \\
= & -[124][136] \\
= & {[134][234]} \\
= & -[123][246]\end{array}$ & $\underline{7}$ & $\underline{[123][234]}(-[124][136][234]+[123][134][246])$ \\
\hline $\begin{array}{l}{[136]} \\
{[246]}\end{array}$ & $\begin{array}{ll}= & -[\mathbf{1 2 3}][\mathbf{1 3 4}] \\
= & -[\mathbf{1 2 4}][\mathbf{2 3 4}]\end{array}$ & $\underline{\underline{6}}$ & 0. \\
\hline
\end{tabular}

Nondegeneracy condition: none.

Example 11. [Nehring's theorem, see also Chou et.al. (1994), Example 6.27]

Free points: 1, 2, 3, 4 .

Semifree point: 5 on $\mathbf{1 2}$.

Intersections:

$$
\begin{array}{llll}
6=12 \cap 34, & 7=13 \cap 24, & 8=23 \cap 14, & 9=13 \cap 58, \\
0=23 \cap 69, & \mathrm{~A}=12 \cap 70, & \mathrm{~B}=13 \cap 8 \mathrm{~A}, & \mathrm{C}=23 \cap 6 \mathrm{~B} .
\end{array}
$$

Conclusion: 5, 7, $\mathrm{C}$ are collinear. 
Fig. 11. Example 11.

Proof:

\begin{tabular}{|c|c|c|c|}
\hline \multicolumn{2}{|r|}{ Rules } & & {$[57 \mathrm{C}]$} \\
\hline $\begin{array}{l}{[67 \mathrm{~B}]} \\
{[56 \mathrm{~B}]}\end{array}$ & $\begin{array}{l}=[136][\mathbf{7 8 A}] \\
=[13 \mathrm{~A}][568]\end{array}$ & $\underline{\mathrm{B}}$ & $-[136][235][78 \mathrm{~A}]-[13 \mathrm{~A}][237][568]$ \\
\hline $\begin{array}{l}{[\mathbf{7 8 A}]} \\
{[\mathbf{1 3 A}]}\end{array}$ & $\begin{array}{l}=-[\mathbf{1 2 7}][\mathbf{7 8 0}] \\
=-[\mathbf{1 2 3}][\mathbf{1 7 0}]\end{array}$ & $\underline{\underline{A}}$ & {$[127][136][235][780]+[123][170][237][568]$} \\
\hline $\begin{array}{l}{[\mathbf{7 8 0}]} \\
{[\mathbf{1 7 0}]}\end{array}$ & $\begin{aligned}= & -[\mathbf{2 3 7}][\mathbf{6 8 9}] \\
= & {[\mathbf{1 2 7}][\mathbf{3 6 9}] }\end{aligned}$ & $\underline{\mathbf{0}}$ & $\underline{[\mathbf{1 2 7}][237]}(-[\mathbf{1 3 6}][\mathbf{2 3 5}][689]+[\mathbf{1 2 3}][\mathbf{3 6 9}][568])$ \\
\hline $\begin{array}{l}{[689]} \\
{[369]}\end{array}$ & $\begin{array}{l}=\quad[\mathbf{1 3 8}][\mathbf{5 6 8}] \\
=-[\mathbf{1 3 6}][\mathbf{3 5 8}]\end{array}$ & $\underline{9}$ & $\underline{[136][568]}(-[138][235]-[123][358])$ \\
\hline $\begin{array}{l}{[138]} \\
{[358}\end{array}$ & $\begin{array}{l}=-[123][134] \\
=\quad[134][235]\end{array}$ & $\underline{\underline{8}}$ & 0. \\
\hline
\end{tabular}

Nondegeneracy condition: none.

Example 12. [See also Richter-Gebert (1995), Example 7]

Free points: $1,2,3,4,5,6,7,8,9$.

Semifree point: $\mathbf{0}$ on $\mathbf{1 9}$.

Intersections:

$$
\begin{array}{llll}
\mathrm{A}=13 \cap 24, & \mathrm{~B}=\mathbf{2 4} \cap 35, & \mathrm{C}=35 \cap 46, & \mathrm{D}=46 \cap 57, \\
\mathrm{E}=57 \cap 68, & \mathrm{~F}=68 \cap 17, & \mathrm{G}=17 \cap 28, & \mathbf{H}=\mathbf{2 8} \cap \mathbf{1 3}, \\
\mathrm{A}_{1}=\mathbf{2 9} \cap \mathbf{0 H}, & \mathrm{B}_{1}=39 \cap \mathrm{AA}_{1}, & \mathrm{C}_{1}=49 \cap \mathrm{BB}_{1}, & \mathrm{D}_{1}=59 \cap \mathrm{CC}_{1}, \\
\mathrm{E}_{1}=\mathbf{6 9} \cap \mathrm{DD}_{1}, & \mathrm{~F}_{1}=\mathbf{7 9} \cap \mathrm{EE}_{1}, & \mathrm{G}_{1}=89 \cap \mathrm{FF}_{1} . &
\end{array}
$$

Conclusion: $\mathbf{0}, \mathbf{G}, \mathbf{G}_{1}$ are collinear. 
Fig. 12. Example 12.

Proof:

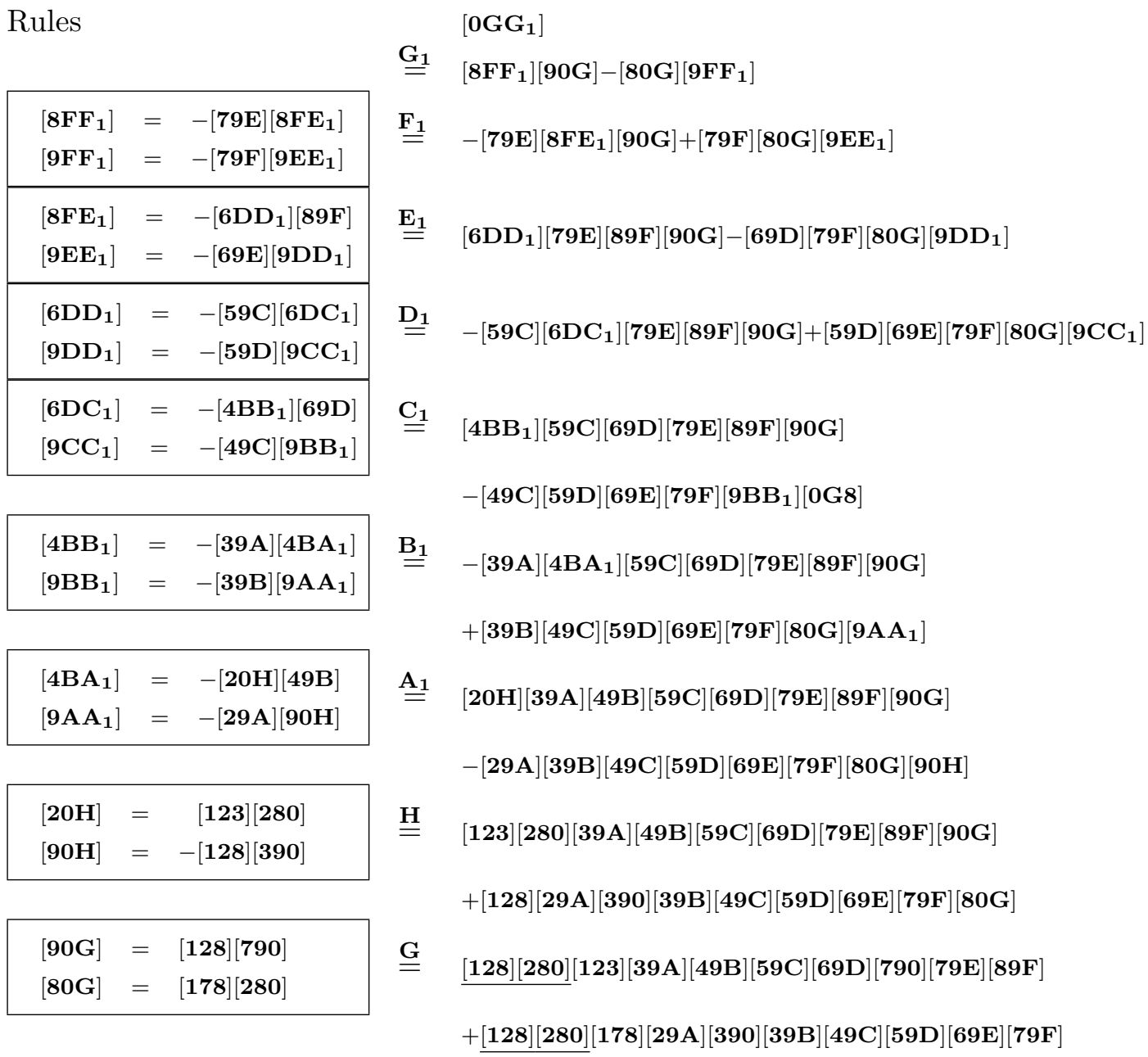




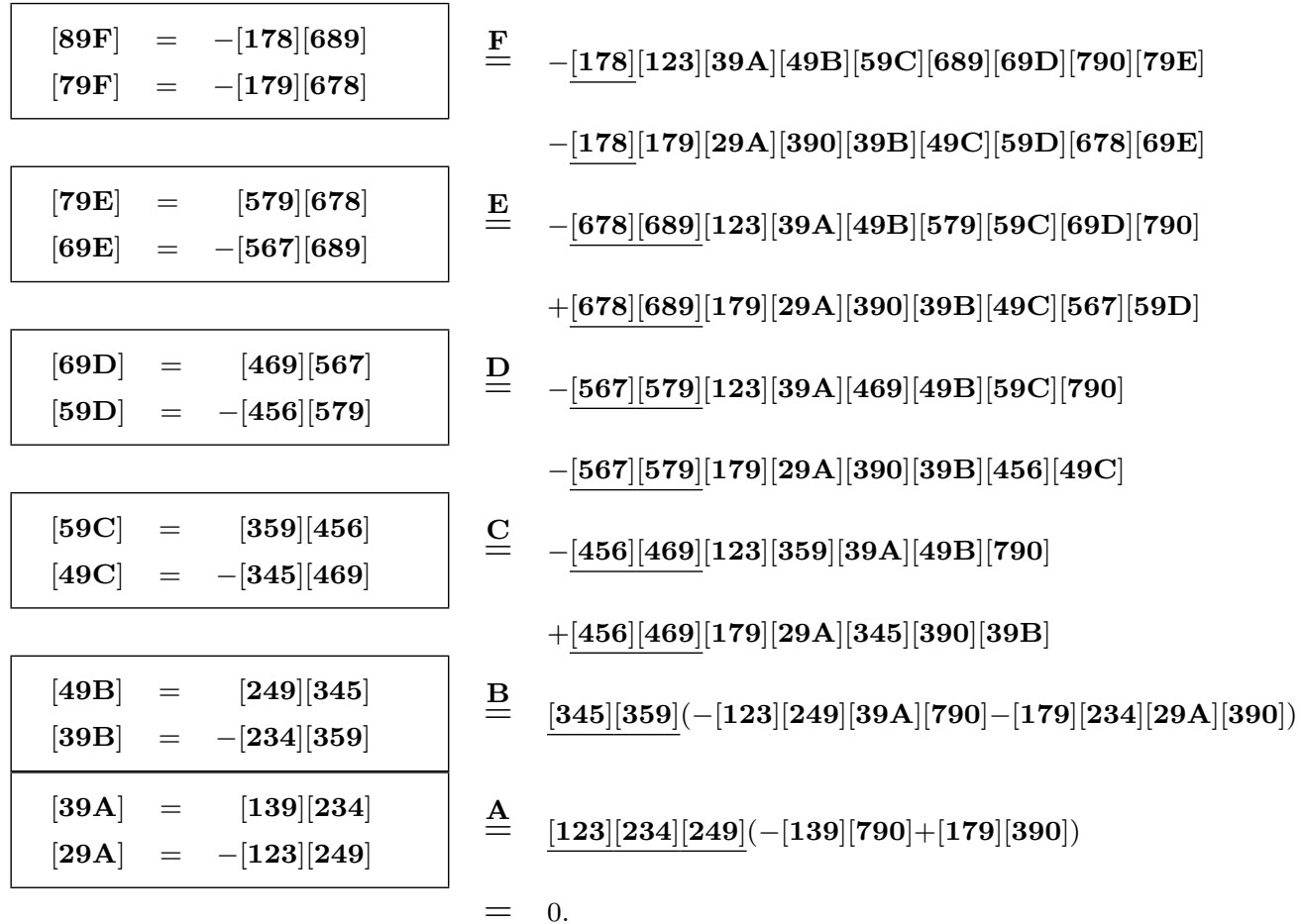

Nondegeneracy condition: none.

Example 13. [Saam's theorem, see also Richter-Gebert (1995), Example 6]

Free points: $1,2,3,4,5,6$.

Semifree point: $\mathbf{7}$ on $\mathbf{1 2}$.

Intersections:

$$
\begin{array}{lll}
8=13 \cap 24, & 9=23 \cap 14, & 0=15 \cap 46, \\
A=35 \cap 16, & \mathrm{~B}=13 \cap 67, & \mathrm{C}=16 \cap 90, \\
\mathrm{D}=15 \cap 8 \mathrm{~A}, & \mathrm{E}=12 \cap \mathrm{BC}, & \mathrm{F}=57 \cap 14 .
\end{array}
$$

Conclusion: $\mathbf{D}, \mathbf{E}, \mathbf{F}$ are collinear.

Fig. 13. Example 13. 
Proof:

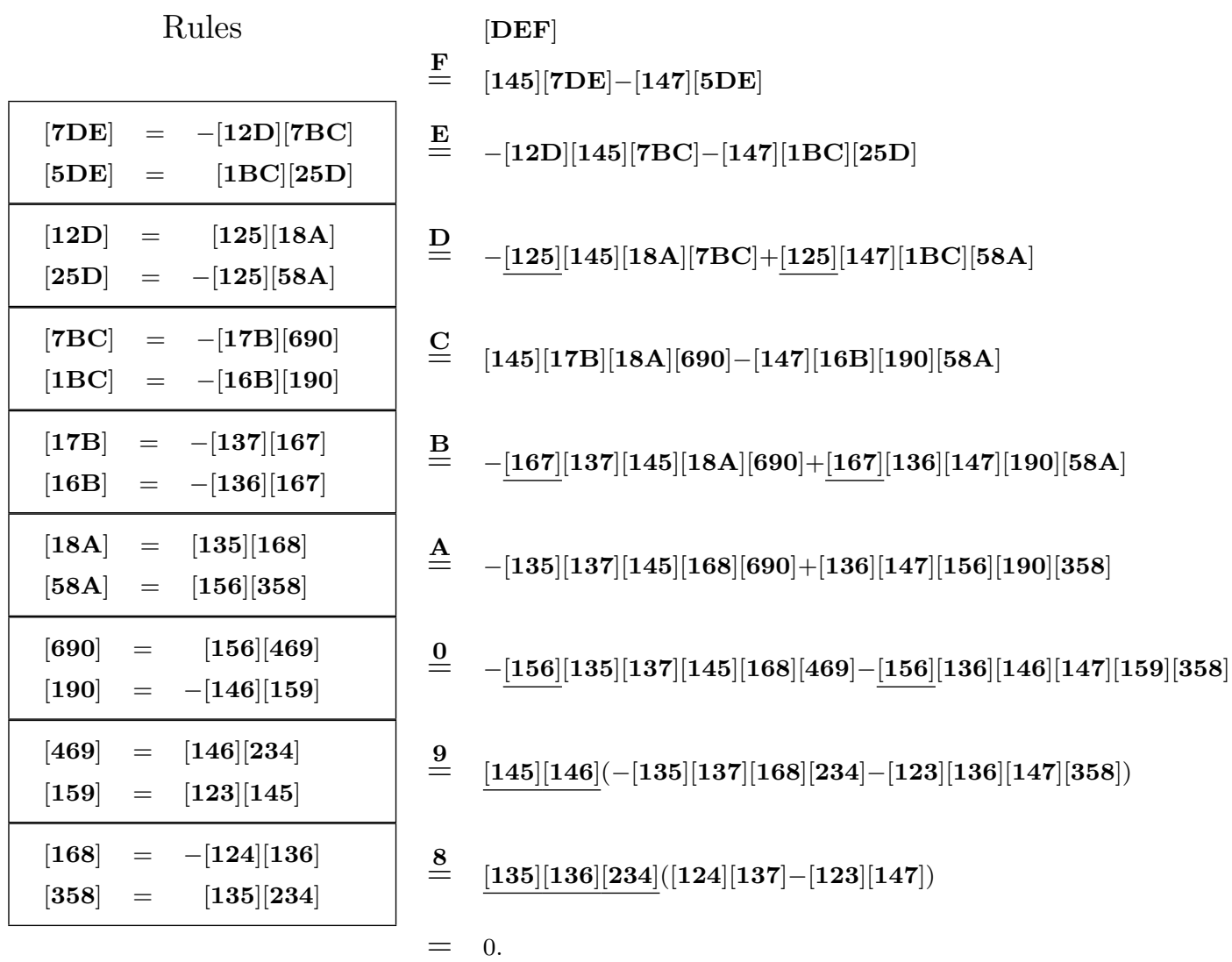

Nondegeneracy condition: none.

Example 14. [See also Chou et. al. (1994), Example 6.190] Two doubly perspective triangles are also triply perspective.

Free points: $\mathbf{1}, \mathbf{2}, \mathbf{3}, \mathbf{4}, \mathbf{5}$.

Intersections: $6=12 \cap 34, \quad 7=24 \cap 15, \quad 8=13 \cap 45, \quad 9=56 \cap 37$.

Conclusion: 2, 8, 9 are collinear.

Fig. 14. Example 14. 
Proof:

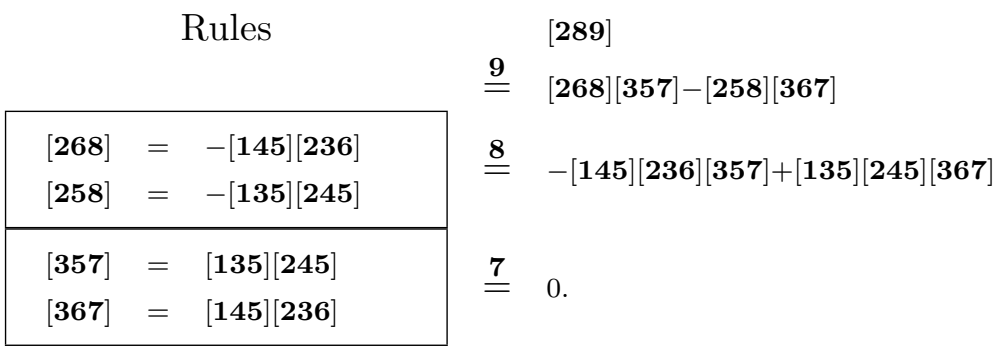

Nondegeneracy condition: none.

Example 15. [See also Chou et. al. (1994), Example 6.26] In a hexagon whose vertices are $1,2,3,4,5,9$, if both $39,12,45$ and 19, 34, 25 are concurrent, then 14, 59, 23 are concurrent.

Free points: $\mathbf{1}, \mathbf{2}, \mathbf{3}, \mathbf{4}, \mathbf{5}$.

Intersections: $6=23 \cap 14, \quad 7=12 \cap 45, \quad 8=34 \cap 25, \quad 9=37 \cap 18$.

Conclusion: 5, 6, 9 are collinear.

Fig. 15. Example 15.

Proof:

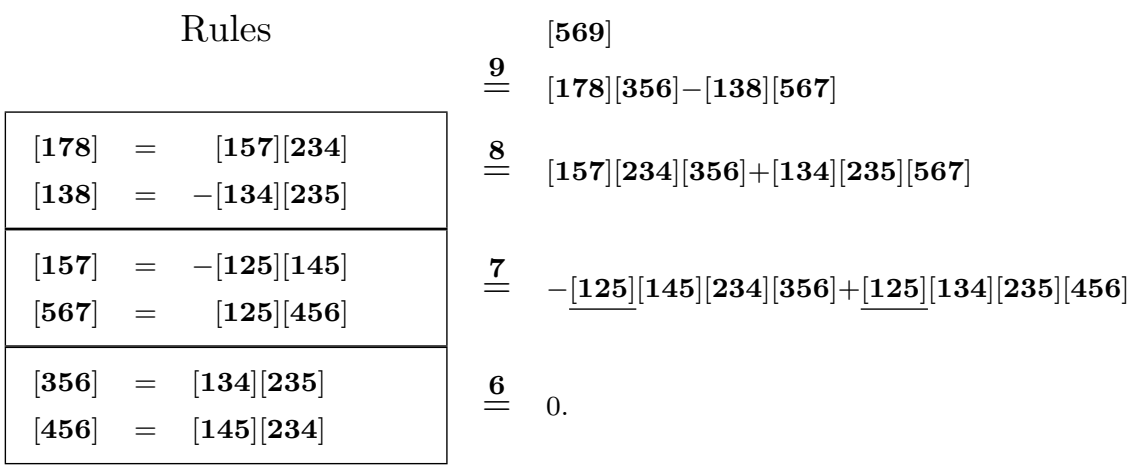

Nondegeneracy condition: none.

Example 16. [Permutation Theorem, see also Richter-Gebert (1995), Example 3] If 6, 7, $\mathbf{8}, \mathbf{9}$ are collinear, then there exits a projectivity between $(\mathbf{8}, \mathbf{9}, \mathbf{7}, \mathbf{6})$ and $(\mathbf{6}, \mathbf{7}, \mathbf{9}, \mathbf{8})$. 
Free points: $1,2,3,4$.

Semifree point: $\mathbf{5}$ on $\mathbf{2 3}$.

Intersections:

$$
6=12 \cap 34, \quad 7=13 \cap 24, \quad 8=15 \cap 67, \quad 9=45 \cap 67, \quad 0=23 \cap 48 .
$$

Conclusion: 1, 9, $\mathbf{0}$ are collinear.

Fig. 16. Example 16.

Proof:

\begin{tabular}{|c|c|c|c|}
\hline \multicolumn{2}{|r|}{ Rules } & - & [190] \\
\hline $\begin{array}{l}{[189]} \\
{[149]}\end{array}$ & $\begin{array}{l}=[148][567] \\
=[145][467]\end{array}$ & $\underline{\underline{9}}$ & {$[148][234][567]-[145][238][467]$} \\
\hline $\begin{array}{l}{[148]} \\
{[238]}\end{array}$ & $\begin{array}{lr}= & {[145][167]} \\
= & -[123][567]\end{array}$ & $\underline{\underline{8}}$ & $\underline{[145][567]}([167][234]+[123][467])$ \\
\hline $\begin{array}{l}{[167]} \\
{[467]}\end{array}$ & $\begin{array}{rr}= & -[124][136] \\
= & {[134][246]}\end{array}$ & $\stackrel{7}{=}$ & $-[124][136][234]+[123][134][246]$ \\
\hline $\begin{array}{l}{[136]} \\
{[246]}\end{array}$ & $\begin{array}{ll}= & -[\mathbf{1 2 3}][\mathbf{1 3 4}] \\
= & -[\mathbf{1 2 4}][\mathbf{2 3 4}]\end{array}$ & $\underline{\underline{6}}$ & 0. \\
\hline
\end{tabular}

Nondegeneracy condition: none.

Example 17. [Harmonic points, see also Chou et. al. (1994), Example 6.236, and RichterGebert (1995), Example 4] If $\mathbf{6}, \mathbf{7}, \mathbf{8}, \mathbf{B}$ is a harmonic quadruple of points, then $\mathbf{B}$ is uniquely determined by $\mathbf{6}, \mathbf{7}, \mathbf{8}$.

Free points: 1, 2, 3, 4, 5 .

Semifree point: $\mathbf{9}$ on $\mathbf{5 8}$.

Intersections:

$$
\begin{array}{lll}
6=12 \cap 34, & 7=23 \cap 14, & 8=67 \cap 13, \\
0=79 \cap 56, & A=69 \cap 57, & \mathrm{~B}=67 \cap 24 .
\end{array}
$$

Conclusion: 0, A, $\mathbf{B}$ are collinear. 
Fig. 17. Example 17.

Proof:

\begin{tabular}{|c|c|c|c|}
\hline \multicolumn{2}{|r|}{ Rules } & & {$[\mathbf{0 A B}]$} \\
\hline$[\mathrm{F0A}]$ & $\begin{array}{lr}= & -[570][679] \\
= & {[567][690]}\end{array}$ & $\underline{\underline{\mathbf{A}}}$ & $-[246][570][679]-[247][567][690]$ \\
\hline $\begin{array}{l}{[570]} \\
{[690]}\end{array}$ & $\begin{array}{l}=[567][579] \\
=[569][679]\end{array}$ & $\underline{\underline{0}}$ & {$[567][679](-[246][579]-[247][569])$} \\
\hline $\begin{array}{l}{[158][5} \\
{[158][5}\end{array}$ & $\begin{array}{l}79]=[159][578] \\
69]=[159][568]\end{array}$ & $\underline{\underline{9}}$ & $\frac{\underline{159]}]}{[158]}(-[246][578]-[247][568])$ \\
\hline $\begin{array}{l}{[578]} \\
{[568]}\end{array}$ & $\begin{array}{l}=[137][567] \\
=[136][567]\end{array}$ & $\underline{\underline{8}}$ & $-\underline{[567]}[137][246]-\underline{[567]}[136][247]$ \\
\hline $\begin{array}{l}{[137]} \\
{[247]}\end{array}$ & $\begin{array}{rr}= & -[123][134] \\
= & {[124][234]}\end{array}$ & $\stackrel{7}{=}$ & {$[123][134][246]-[124][136][234]$} \\
\hline $\begin{array}{l}{[246]} \\
{[136]}\end{array}$ & $\begin{array}{l}=-[\mathbf{1 2 4}][\mathbf{2 3 4}] \\
=-[\mathbf{1 2 3}][\mathbf{1 3 4}]\end{array}$ & $\underline{\underline{6}}$ & 0. \\
\hline
\end{tabular}

Nondegeneracy condition: $[\mathbf{1 5 8}] \neq 0$.

Example 18. [See also Chou et. al. (1994), Example 6.237, and Richter-Gebert (1995), Example 5] If the intersections of five correponding sides of two complete quadrilaterals are on the same line $l$, then the remaining sides also meet in $l$.

Free points: $1,2,3,4,5,6$.

Semifree point: $\mathbf{7}$ on $\mathbf{1 2}$.

Intersections:

$$
\begin{array}{llll}
8=23 \cap 56, & 9=13 \cap 78, & 0=14 \cap 78, & A=24 \cap 78 \\
B=34 \cap 78, & C=57 \cap 69, & D=5 A \cap 6 B .
\end{array}
$$

Conclusion: $\mathbf{0}, \mathbf{C}, \mathbf{D}$ are collinear. 
Fig. 18. Example 18.

Proof:

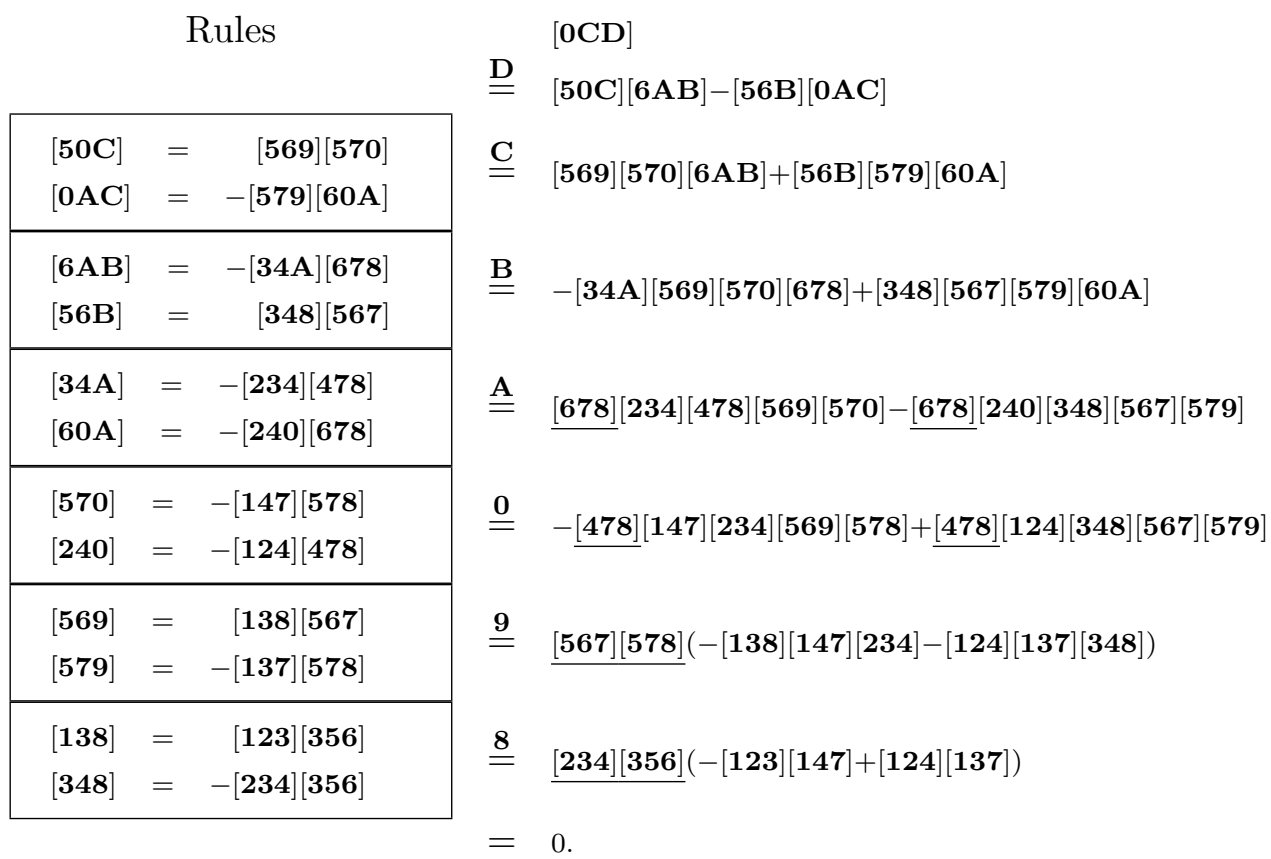

Nondegeneracy condition: none.

Example 19. [Pascal's theorem, see also Chou et al. (1994), Example 6.390]

Free points: $\mathbf{1}, \mathbf{2}, \mathbf{3}, \mathbf{4}, \mathbf{5}$.

Semifree point: $\mathbf{6}$ on $\mathbf{1 2}$.

Intersections:

$$
\begin{array}{lll}
7=34 \cap 15, & 8=46 \cap 59, & 2=16 \cap 39, \\
\mathrm{~A}=36 \cap 15, & \mathrm{~B}=45 \cap 69, & 0=34 \cap 19 .
\end{array}
$$

Conclusion: If $\mathbf{2 ,}, \mathbf{7}, \mathbf{8}$ are collinear, so are $\mathbf{0}, \mathbf{A}, \mathbf{B}$.

Reformulation of the theorem:

Free points: $\mathbf{1 , 2}, \mathbf{3}, \mathbf{4}, \mathbf{5}$.

Semifree point: $\mathbf{6}$ on $\mathbf{1 2}$. 
Intersections:

$$
\begin{aligned}
& 7=15 \cap 34, \quad 8=27 \cap 46, \quad 9=58 \cap 23, \\
& 0=19 \cap 34, \quad A=36 \cap 15, \quad B=69 \cap 45 .
\end{aligned}
$$

Conclusion: $\mathbf{0}, \mathbf{A}, \mathbf{B}$ are collinear.

Fig. 19. Example 19.

Proof:

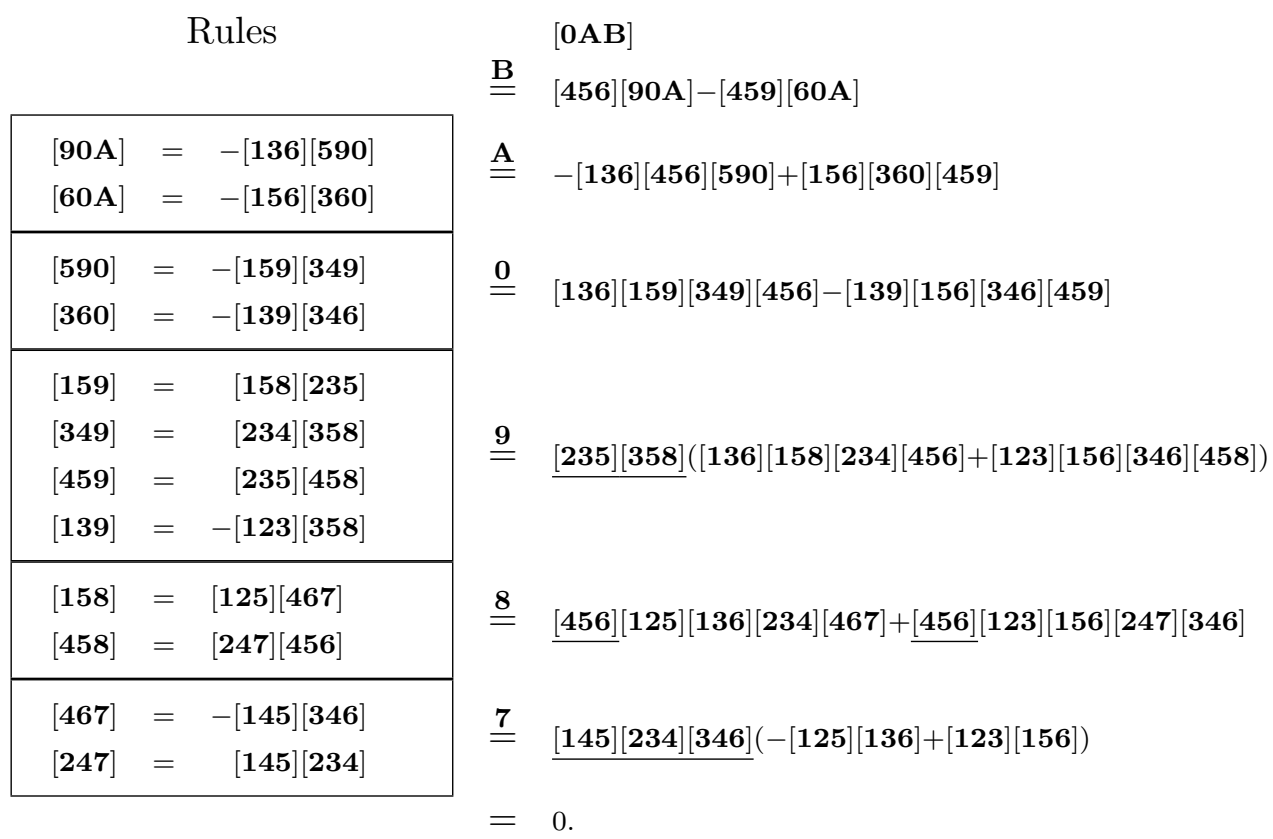

Nondegeneracy condition in the proof of the reformulated theorem: none.

Example 20. [See also Chou et. al. (1994), Example 6.28]

Free points: 1, 2, 3, 4, 5, 6 .

Semifree points: $\mathbf{7}$ on $\mathbf{1 2}, \mathbf{8}$ on $\mathbf{1 3}$.

Intersections:

$$
\begin{array}{lll}
9=14 \cap 56, & \mathbf{0}=15 \cap 46, & \mathrm{~A}=37 \cap 28, \\
\mathrm{~B}=34 \cap 89, & \mathrm{C}=\mathbf{2 5} \cap 70, & \mathrm{D}=\mathbf{5 8} \cap \mathbf{3 0} .
\end{array}
$$


Fig. 20. Example 20.

Conclusion: A, C, D are collinear.

Proof:

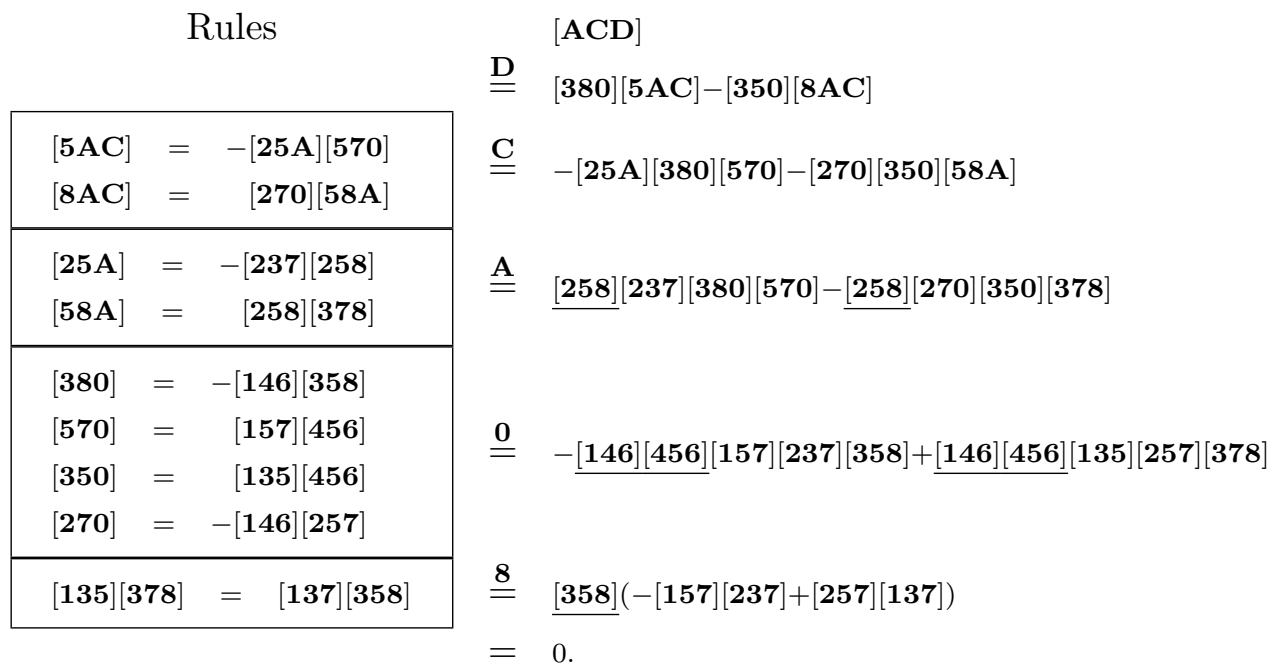

Nondegeneracy condition: $[\mathbf{1 3 5}] \neq 0$.

Example 21. [See also Chou et. al. (1994), Example 6.33]

Free points: $3,4,6,7$.

Intersections: $1=36 \cap 47, \quad 2=46 \cap 37,8=67 \cap 34$.

Semifree points: $\mathbf{9}$ on $\mathbf{1 8}, \mathbf{0}$ on $\mathbf{1 2}$.

Intersections: $A=\mathbf{2 8} \cap \mathbf{9 0}, \quad B=36 \cap 7 A, \quad C=67 \cap 39, \quad 5=37 \cap 60$.

Conclusion: $\mathbf{B}, \mathbf{C}, \mathbf{5}$ are collinear.

Reformulation of the theorem:

Free points: 1, 2, 3, 4 .

Semifree points: $\mathbf{5}$ on $\mathbf{2 3}, \mathbf{9}$ on $\mathbf{1 2 .}$

Intersections:

$$
\begin{array}{llll}
6=13 \cap 24, & 7=23 \cap 14, & 8=34 \cap 67, & 0=56 \cap 18, \\
A=28 \cap 90, & B=7 A \cap 13, & C=39 \cap 67 .
\end{array}
$$


Conclusion: 5, B, C are collinear.

Fig. 21. Example 21.

Proof:



Nondegeneracy condition in the proof of the reformulated theorem: $[\mathbf{1 2 3}] \neq 0$.

Example 22. [Non-realizable $10_{3}$-configuration, see also Richter-Gebert (1995), Example 9]

Free points: $1,2,3,4,5$.

Semifree point: $\mathbf{6}$ on $\mathbf{1 2}$.

Intersections: $7=23 \cap 14, \quad 8=15 \cap 46, \quad 9=25 \cap 36, \quad 0=34 \cap 57$.

Conclusion: 8, 9, $\mathbf{0}$ are not collinear. 
Fig. 22. Example 22.

Proof:

\begin{tabular}{|c|c|c|c|c|}
\hline \multicolumn{3}{|c|}{ Rules } & \multicolumn{2}{|r|}{$[890]$} \\
\hline $\begin{array}{l}{[489]} \\
{[389]}\end{array}$ & & $\begin{array}{r}{[256][348]} \\
-[235][368]\end{array}$ & $\underline{\underline{9}}$ & {$[256][348][357]+[235][368][457]$} \\
\hline $\begin{array}{l}{[348]} \\
{[368]}\end{array}$ & & $\begin{array}{r}{[145][346]} \\
-[156][346]\end{array}$ & $\underline{\underline{8}}$ & $\underline{[346]}[145][256][357]-\underline{3466][156][235][457]}$ \\
\hline $\begin{array}{l}{[357]} \\
{[457]}\end{array}$ & & $\begin{array}{l}{[134][235]} \\
{[145][234]}\end{array}$ & $\stackrel{7}{=}$ & $\underline{[145][235][346]([134][256]-[156][234])}$ \\
\hline$[123][2$ & & $=[\mathbf{1 2 5}][236]$ & $\underline{6}$ & $\underline{\underline{\underline{145}][235][346][125]}}([134][236]-[136][234])$ \\
\hline$[123][1$ & & $=[125][136]$ & & $\frac{[123]}{[125][145][235][346]^{2}}$ \\
\hline
\end{tabular}

Nondegeneracy conditions: $[\mathbf{1 2 5}],[\mathbf{1 4 5}],[\mathbf{2 3 5}],[\mathbf{3 4 6}] \neq 0$.

Example 23. [Fano's axiom, see also Kadison and Kromann (1996), p. 46, and O'hara and Ward (1936), p. 66 ] There is no complete quadrilateral whose three diagonal points are collinear.

Free points: $\mathbf{1}, \mathbf{2}, \mathbf{3}, \mathbf{4}$.

Intersections: $\mathbf{5}=\mathbf{1 2} \cap \mathbf{3 4}, \mathbf{6}=\mathbf{2 3} \cap \mathbf{1 4}, \mathbf{7}=\mathbf{1 3} \cap \mathbf{2 4}$.

Conclusion : $\mathbf{5 , 6}, \mathbf{7}$ are not collinear.

Proof:

\begin{tabular}{|c|c|c|c|}
\hline \multicolumn{2}{|c|}{ Rules } & \multirow{2}{*}{$\begin{array}{l}\underline{7} \\
\underline{\underline{6}}\end{array}$} & {$[567]$} \\
\hline$[356]$ & $=[134][235]$ & & {$[124][134][255]+[123][145][234$} \\
\hline [156] & $=[123][145]$ & & {$[124][104][200]+[120][140][204]$} \\
\hline$[235]$ & $=-[123][234]$ & $\underline{\underline{5}}$ & $-2[\mathbf{1 2 3}][\mathbf{1 2 4}][\mathbf{1 3 4}][\mathbf{2 3 4}]$. \\
\hline [145] & $=-[\mathbf{1 2 4}][\mathbf{1 3 4}]$ & & \\
\hline
\end{tabular}

Nondegeneracy conditions: $[\mathbf{1 2 3}],[\mathbf{1 2 4}],[\mathbf{1 3 4}],[\mathbf{2 3 4}] \neq 0$. 
Fig. 23. Example 23.

\section{References}

[1] J. Bokowski and J. Richter-Gebert. On the finding of final polynomials, Europ. J. Combinatorics 11, 21-34, 1990.

[2] B. Buchberger. Gröbner Basis-an Algorithmic Method in Polynomial Ideal Theory, in: Multidimensional Systems Theory, N. K. Bose (ed.). 1985.

[3] S.-C. Chou, X.-S. Gao and J.-Z. Zhang. Machine Proofs in Geometry-Automated Production of Readable Proofs for Geometric Theorems. World Scientific, 1994.

[4] H. Crapo and J. Richter-Gebert. Automatic Proving of Geometric Theorems, in: Invariant Methods in Discrete and Computational Geometry, N. White (ed.), 107-139. 1994.

[5] P. Doubilet, G. C. Rota and J. Stein. On the Foundations of Combinatorial Theory IX: Combinatorial Methods in Invariant Theory, Stud. Appl. Math. 57, 185-216. 1974.

[6] L. Kadison and M. T. Kromann. Projective Geometry and Modern Algebra, Birkhäuser, Boston, 1996.

[7] H. Li and M.-T. Cheng. Proving theorems in elementary geometry with Clifford algebraic method. Chinese Math. Progress 26(4): 357-371. 1997.

[8] H. Li. Vectorial equation-solving for mechanical geometry theorem proving. J. Automated Reasoning 25, 83-121. 2000.

[9] H. Li and Y. Wu. Outer Product Factorization in Clifford Algebra, Proc. ATCM99, pp. 255-264, Guangzhou. 1999.

[10] B. Mourrain and N. Stolfi. Computational Symbolic geometry, in: Invariant Methods in Discrete and Computational Geometry, N. White (ed.), pp. 107-139. 1994.

[11] C. W. O'hara, S. J. and D. R. Ward, S. J. An Introduction to Projective Geometry, Oxford University Press, London, 1936.

[12] D. Pedoe. An Introduction to Projective Geometry, Pergamon Press, Oxford, 1963.

[13] J. Richter-Gebert. Mechanical Theorem Proving in Projective Geometry, Annals of Math. and Artificial Intelligence 13, 159-171. 1995.

[14] B. Sturmfels. Computing final polynomials and final syzygies using Buchberger's Gröbner bases method, Result. Math. 15, 351-360. 1989.

[15] B. Sturmfels. Algorithms in Invariant Theory. Springer, New York, 1993.

[16] B. Sturmfels and N. White. Gröbner bases and invariant theory, Adv. Math. 76, 245-259. 1989.

[17] D. Wang. Elimination procedures for mechanical theorem proving in geometry, Annals of Math. and Artificial Intelligence 13, 1-24. 1995. 
[18] B. Sturmfels and W. Whitely. On the Synthetic Factorization of Homogeneous Invariants, $J$. Symbolic Computation 11, 439-454. 1991.

[19] W.-T. Wu. On the Decision Problem and the Mechanization of Theorem Proving in Elementary Geometry, Contemp. Math. 29, 213-234, 1984.

[20] W.-T. Wu. Basic Principles of Mechanical Theorem Proving in Elementary Geometries, J. Sys. Sci. \& Math. Sci. 4, 207-235. 1984.

[21] A. Young. On quantative substitutionals analysis (3rd paper), Proc. London Math. Soc., Ser. 2, 28, 255-292, 1928. 